\title{
FATORES QUE INFLUENCIAM NA QUALIDADE DE VIDA DE IDOSOS COM DOENÇA DE ALZHEIMER
}

\footnotetext{
Aline Sharlon Maciel Batista Ramos ${ }^{1}$; Adriana Alves Reis ${ }^{2}$; Débora Luana Ribeiro

Pessoa ${ }^{3}$; Hariane Freitas Rocha Almeida ${ }^{4}$; Roseane Rodrigues Mendes Costa ${ }^{5}$.

${ }^{1}$ Enfermeira. Doutoranda em Ciências- UERJ. Professora Assistente da Universidade CEUMA. E-mail: alinesharlon@gmail.com

${ }^{2}$ Discente do 5ำ ano do Curso de Enfermagem da Universidade CEUMA. E-mail: adryalves@hotmail.com

${ }^{3}$ Farmacêutica. Doutorado em Biotecnologia. Professora Adjunta da Universidade Federal do Maranhão (UFMA).

${ }^{4}$ Discente do $5^{\circ}$ ano do Curso de Enfermagem da Universidade CEUMA.

${ }^{5}$ Enfermeira. Especialista em Dermoestética e Cosmética Aplicada pela Universidade Ceuma (Uniceuma). Especialista em Gerontologia pelo Instituto de Estudos Superiores do Maranhão (IESMA). Especialista em Formação Pedagógica em Educação Profissional pela Universidade Federal do Maranhão (UFMA). Graduada em Enfermagem pela Universidade

Federal do Maranhão (UFMA).
}

\section{Recebido em: 06/04/2018 - Aprovado em: 10/06/2018 - Publicado em: 20/06/2018}

DOI: 10.18677/EnciBio_2018A124

\begin{abstract}
RESUMO
A Doença de Alzheimer (DA) é um transtorno neurodegenerativo progressivo que se manifesta por deterioração cognitiva e da memória, comprometimento progressivo das atividades de vida diária e uma variedade de sintomas neuropsiquiátricos e de alterações comportamentais. Tratou-se de uma revisão integrativa (RI) da literatura, realizada de acordo com seis passos operacionais: identificação do problema; elaboração da pergunta norteadora; definição de critérios de inclusão e exclusão; coleta de dados em bases científicas; seleção dos artigos; análise e interpretação dos resultados. Partiu-se do seguinte questionamento: quais os fatores que influenciam na qualidade de vida de idosos com Doença de Alzheimer? Foram realizadas buscas nas bases de dados: PubMED e Scientific Electronic Library Online (SciELO), sendo selecionadas as publicações com o texto completo, redigidas em português e que abordaram "Qualidadade de Vida (quality of life)", "Idosos (aged)", "Alzheimer (alzheimer disease)" publicados no período de 2006 a 2016. A análise dos artigos evidenciou que 0 bom suporte familiar e socioeconômico, junto à prática de exercícios físicos, tarefas e atividades de lazer, participação em programas assistenciais multidisciplinares de reabilitação cognitiva e neuropsicológica que envolva o paciente e o cuidador, a arteterapia e as técnicas de reminiscência favorecem o bem-estar e melhoram a qualidade de vida de idosos com DA. O enfermeiro precisa conhecer os fatores que influenciam na qualidade de vida do idoso com DA para que, junto à equipe multidisciplinar, realize atividades complementares ao tratamento farmacológico, que valorizem as necessidades de cada paciente e contribuam para a minimização das incapacidades provocadas pela doença.
\end{abstract}

Descritores: Alzheimer; Idosos; Qualidade de vida. 


\section{FACTORS THAT INFLUENCE THE QUALITY OF LIFE OF ELDERLY PEOPLE WITH ALZHEIMER'S DISEASE ABSTRACT}

Alzheimer's disease (AD) is a progressive neurodegenerative disorder manifested by cognitive and memory deterioration, progressive impairment of daily living activities, and a variety of neuropsychiatric symptoms and behavioral changes. It was an integrative review (IR) of the literature, carried out according to six operational steps: identification of the issue; guiding question formulation; definition of inclusion and exclusion criteria; collection of data on scientific bases; selection of articles; analysis and interpretation of results. The following question was asked: what factors influence the quality of life of elderly people affected by Alzheimer's disease? It was searched the databases: PubMED and Scientific Electronic Library Online (SciELO), selecting the publications with the complete text, written in Portuguese and that approched "Quality of life", "Elderly people", and "Alzheimer's disease" published between 2006 and 2016. The analysis of the articles demonstrated that good family and socioeconomic support, in addition to the practice of physical exercises, tasks and leisure activities, participation in multidisciplinary care programs of cognitive rehabilitation and neuropsychological treatment that involves the patient and the caregiver, art therapy and reminiscence techniques favor well-being and improve the quality of life of elderly patients with $A D$. The nurse needs to know the factors that influence the quality of life of the elderly with $A D$ so that, along the multidisciplinary team, perform complementary activities to the pharmacological treatment, that value the needs of each patient and contribute to the minimization of the incapacities caused by the disease.

Keywords: Quality of life; Aged; Alzheimer disease.

\section{INTRODUÇÃO}

Considerado um processo heterogêneo, o envelhecimento diferenciase de indivíduo para indivíduo, apresentando características individuais que o divide em dois conceitos, o biológico, no qual envelhecer se relaciona à genética e o psíquico, que se associa a relações e dimensões sociais em que este indivíduo está inserido (FECHINI; TROMPIERI, 2016).

Sob este prisma, a OMS (2017) esclarece que em todo o mundo há cerca de 50 milhões de pessoas que sofrem de demência e, a cada ano, cerca de 10 milhões de novos casos são registrados. A doença de Alzheimer(DA), que é a forma mais comum de demência, representa entre $60 \%$ e $70 \%$ dos casos.

A prevalência da demência aumenta progressivamente com 0 envelhecimento, sendo a idade o maior fator de risco para a doença. A partir dos 65 anos, sua prevalência dobra a cada cinco anos, chegando a 5,6\% entre 70 e 79 anos, e 38,6\% nos nonagenários (APRAHAMIAN; MARTINELLI; YASSUDA, 2009).

Segundo a Associação Brasileira de Alzheimer (ABRAz), estima-se que existam cerca de 35,6 milhões de pessoas com DA. No Brasil, há cerca de 1,2 milhão de casos, a maior parte deles ainda sem diagnóstico. A DA é uma enfermidade incurável, causada pela morte de células cerebrais, porém, quando diagnosticada no início, é possível retardar seu avanço e controlar os sintomas (ABRAz, 2017).

Para Talmelli et al. (2013), há uma forte correlação entre o estágio de demência e a capacidade de realizar atividades da vida diária, tornando a avaliação da capacidade funcional tão essencial quanto o diagnóstico, pois se 
refere ao impacto da doença ou da condição limitante do indivíduo, impedindo ou dificultando a realização de suas atividades, refletindo na qualidade de vida (QV) do paciente e de seus familiares com repercussão para o sistema de saúde como um todo.

Diante do exposto, considerando a necessidade de ações que promovam bem estar e contribuam para a QV desta população, o objetivo do presente estudo é realizar uma revisão integrativa de literatura sobre fatores que interferem na qualidade de vida de idosos com Doença de Alzheimer.

MATERIAL E MÉTODOSUtilizou-se a Revisão Integrativa (RI) da Literatura, método de revisão específico que segundo Soares (2014) reúne achados de estudos desenvolvidos mediante diferentes metodologias, permitindo aos revisores sintetizar resultados sem ferir a filiação epistemológica dos estudos empíricos incluídos, e requer que os revisores procedam à análise e à síntese dos dados primários de forma sistemática e rigorosa.

Realizaram-se os seis passos operacionais: identificação do problema; elaboração da pergunta norteadora; definição de critérios de inclusão e exclusão; coleta de dados em bases científicas; seleção dos artigos; análise e interpretação dos resultados.Partiu-se da seguinte pergunta norteadora: quais os fatores que influenciam na qualidade de vida de idosos com Doença de Alzheimer?

A seleção da amostra obedeceu aos seguintes critérios de inclusão: artigos publicados no recorte temporal entre 2006 e 2016, redigidos no idioma português, completos, disponíveis online gratuitamente e que responderam à questão norteadora. Foram excluídas as publicações que não contemplavam o recorte temporal estabelecido, que não estavam redigidas em língua portuguesa, que se encontravam indisponíveis eletronicamente de forma completa e gratuita e que não responderam à questão norteadora desta revisão.

A coleta de dados ocorreu no período de setembro e outubro de 2017 e realizou-se por meio de busca eletrônica utilizando as bases de dados PubMED e Scientific Electronic Library Online (SciELO).

A estratégia de busca utilizada foi realizada por meio da combinação de palavras-chave do MeSH (Medical Subject Heading): "Quality of life"[Mesh] AND "Aged" AND "Alzheimer Disease/Psicology"[Mesh], incluindo os filtros: texto completo grátis, publicados nos últimos dez anos, com o texto em português, e através dos seguintes DeCS (Descritores em Ciências da Saúde): Qualidade de Vida, Idosos e Alzheimer.

Realizou-se a análise sistemática da elegibilidade de cada estudo, inicialmente pelos títulos e depois por resumos e textos completos. Em seguida, foi analisada a qualidade metodológica e, após a exclusão pelos critérios estabelecidos, foi realizada a leitura pormenorizada e a extração dos dados dos artigos selecionados.

\section{RESULTADOS E DISCUSSÃO}

Através da busca eletrônica no PubMED, realizada por meio da combinação das seguintes palavras-chave do MeSH: "Quality of life"[Mesh] AND "Aged" AND "Alzheimer Disease/Psicology"[Mesh], foram localizadas 481 publicações e ao incluir os filtros: texto completo grátis, publicados nos últimos dez anos e com o texto em português, restaram apenas duas publicações, que após análise conforme os critérios de inclusão reduziu-se a uma publicação. 
Por meio da busca eletrônica realizada no SciELO, utilizando os seguintes DeCS: Qualidade de Vida, Idosos e Alzheimer, foram localizadas 14 publicações, onde apenas 10 estavam disponíveis com o texto em português, sendo seis excluídas por não obedecerem aos critérios de inclusão e uma por estar duplicada nas bases de dados utilizadas para esta revisão, resultando em uma amostra de 04 publicações.

Assim, a amostra final desta revisão foi constituída por cinco publicações, sendo uma retirada do PubMED e quatro retiradas da base de dados SciELO. A estratificação da estratégia de busca está representada na Figura 1.

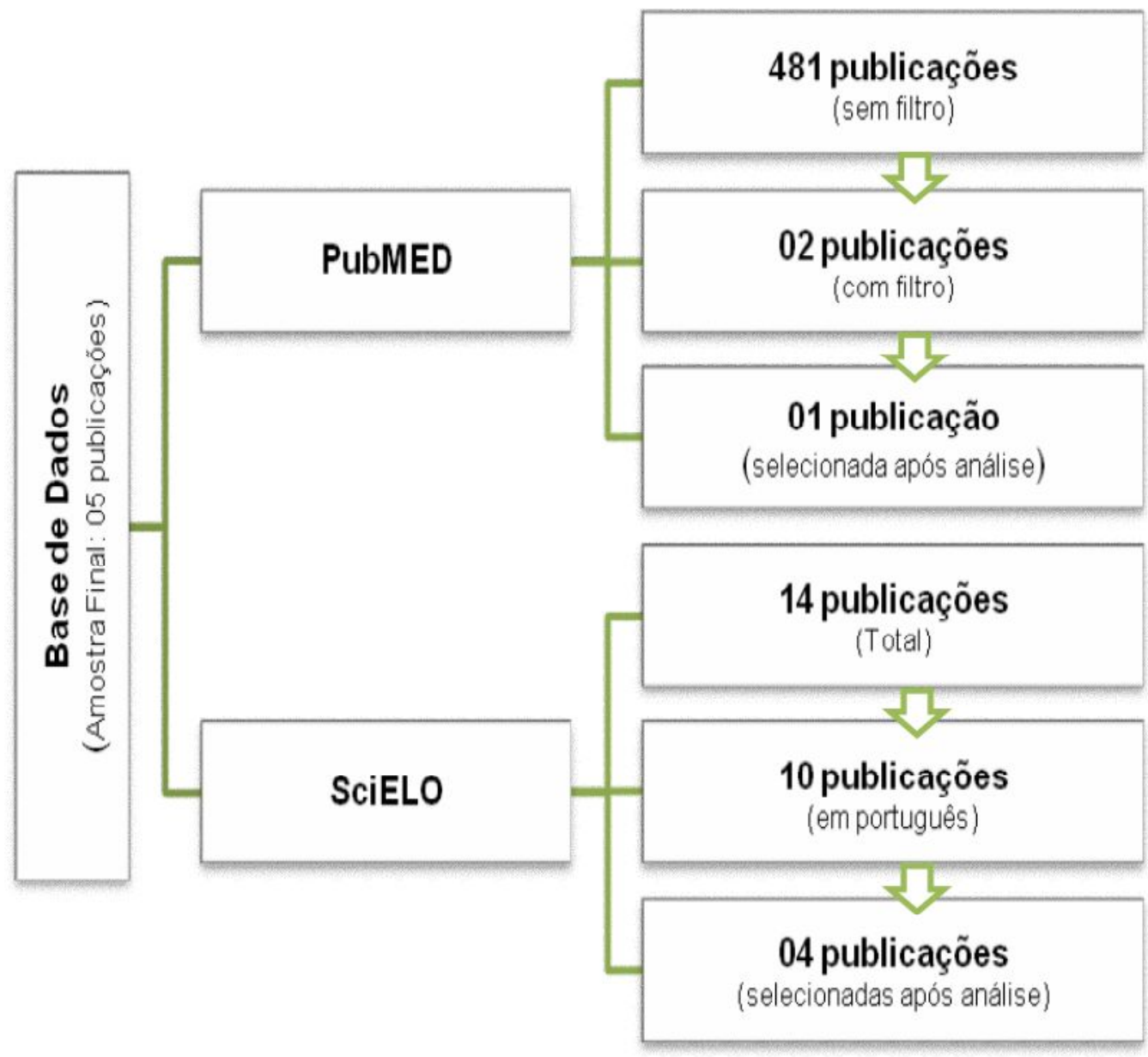

Figura 1 - Estratificação da estratégia de busca.

Após a leitura na íntegra, os estudos selecionados foram identificados por ordem cronológica de E1 a E5. O Quadro 1 caracteriza os conteúdos das publicações quanto à base de dados, título, autor, ano, revista de publicação e objetivos. 
Quadro 1 - Distribuição das publicações selecionadas segundo a base de dados, título, autor, ano, revista de publicação e objetivos.

\begin{tabular}{|c|c|c|c|c|}
\hline Estudo & $\begin{array}{c}\text { Base de } \\
\text { dados }\end{array}$ & Titulo & $\begin{array}{l}\text { Autor, ano e } \\
\text { revista de } \\
\text { publicação }\end{array}$ & Objetivos \\
\hline E1 & PubMED & \begin{tabular}{ll} 
Influência & da \\
doença & de \\
Alzheimer & na \\
percepção & de \\
qualidade & de \\
\multicolumn{2}{c}{ vida do idoso. }
\end{tabular} & $\begin{array}{l}\text { INOUYE; } \\
\text { PEDRAZZANI } \\
\text {; PAVARINI, } \\
\text { 2010. Revista } \\
\text { da Escola de } \\
\text { Enfermagem } \\
\text { da USP. }\end{array}$ & $\begin{array}{l}\text { Comparar a percepção geral } \\
\text { e de cada dimensão de } \\
\text { qualidade de vida (QV) de um } \\
\text { grupo de idosos com Doença } \\
\text { de Alzheimer (DA) com as de } \\
\text { um grupo semelhante quanto } \\
\text { às variáveis } \\
\text { sociodemográficas. }\end{array}$ \\
\hline E2 & SciELO & $\begin{array}{l}\text { Percepções de } \\
\text { suporte familiar } \\
\text { e qualidade de } \\
\text { vida entre } \\
\text { idosos } \\
\text { segundo a } \\
\text { vulnerabilidade } \\
\text { Social. }\end{array}$ & $\begin{array}{l}\text { INOUYE et al. } \\
2010 . \\
\text { Psicologia: } \\
\text { Reflexão e } \\
\text { Crítica. }\end{array}$ & $\begin{array}{l}\text { Examinar a relação entre a } \\
\text { percepção de qualidade de } \\
\text { vida e de suporte familiar } \\
\text { entre idosos, segundo seu } \\
\text { nível de vulnerabilidade social } \\
\text { e características } \\
\text { sociodemográficas. }\end{array}$ \\
\hline E3 & SciELO & $\begin{array}{l}\text { Qualidade de } \\
\text { vida de idosos } \\
\text { com doença de } \\
\text { Alzheimer e de } \\
\text { seus } \\
\text { cuidadores. }\end{array}$ & $\begin{array}{l}\text { BORGHI } \\
\text { et al. } 2011 . \\
\text { Revista } \\
\text { Gaúcha de } \\
\text { Enfermagem }\end{array}$ & $\begin{array}{l}\text { Identificar a qualidade de vida } \\
\text { (QV) do cuidador e a do idoso } \\
\text { com Doença de Alzheimer } \\
\text { (DA). }\end{array}$ \\
\hline E4 & SciELO & $\begin{array}{l}\text { Efeitos de um } \\
\text { programa de } \\
\text { atividade física } \\
\text { sobre os } \\
\text { sintomas } \\
\text { depressivos e } \\
\text { a qualidade de } \\
\text { vida de idosos } \\
\text { com demência } \\
\text { de Alzheimer. }\end{array}$ & $\begin{array}{l}\text { GROPPO } \\
\text { et al. } 2012 . \\
\text { Jornal } \\
\text { Brasileiro de } \\
\text { Psiquiatria }\end{array}$ & $\begin{array}{l}\text { Analisar os efeitos de um } \\
\text { programa de exercícios } \\
\text { físicos sobre os sintomas } \\
\text { depressivos e a percepção da } \\
\text { qualidade de vida (QV) de } \\
\text { pacientes com Doença de } \\
\text { Alzheimer (DA) e de seus } \\
\text { cuidadores. }\end{array}$ \\
\hline E5 & SciELO & $\begin{array}{l}\text { Tratamentos } \\
\text { não } \\
\text { farmacológicos } \\
\text { que melhoram } \\
\text { a qualidade de } \\
\text { vida de idosos } \\
\text { com doença de } \\
\text { Alzheimer: } \\
\text { uma revisão } \\
\text { sistemática. }\end{array}$ & $\begin{array}{l}\text { CARVALHO; } \\
\text { MAGALHÃES } \\
\text {; PEDROSO, } \\
\text { 2016. Revista } \\
\text { Brasileira de } \\
\text { Educação } \\
\text { Física e } \\
\text { Esporte. }\end{array}$ & $\begin{array}{l}\text { Realizar uma revisão } \\
\text { sistemática sobre quais são } \\
\text { os tratamentos não } \\
\text { farmacológicos que ajudam a } \\
\text { melhorar a qualidade de vida } \\
\text { (QV) de idosos com doença } \\
\text { de Alzheimer (DA) mais } \\
\text { descritos na literatura nos } \\
\text { últimos dez anos (2006- } \\
2016) \text {. }\end{array}$ \\
\hline
\end{tabular}

Ao analisar os estudos encontrados, identificou-se que foram poucas as publicações em português realizadas no recorte temporal entre 2006 a 2016. O ano de 2010 destacou-se por apresentar 40\% $(n=2)$ das publicações a cerca da temática proposta. 
Em relação ao tipo de revista de publicação do estudo observou-se que $40 \%(n=2)$ correspondiam à área da Enfermagem, $20 \%(n=1)$ relacionavam-se à área de Psicologia, 20\% $(n=1)$ referiam-se à Educação Física e 20\% $(n=1)$ eram de abordagem Psiquiátrica. Estes resultados evidenciam a complementariedade da equipe multiprofissional no desenvolvimento e prática de ações voltadas à promoção da qualidade de vida de idosos com DA.

No que se refere aos objetivos dos artigos selecionados, percebeu-se que todos de uma forma geral, responderam a pergunta norteadora deste estudo, pois as publicações buscaram comparar, examinar, identificar, analisar e revisar os fatores que influenciam na qualidade de vida de idosos com DA.

O Quadro 2 apresenta a distribuição dos estudos segundo a síntese dos principais resultados e conclusões. Os dados foram agrupados e analisados com caráter descritivo, de modo a responder aos objetivos da pesquisa.

Quadro 2 - Distribuição dos estudos selecionados segundo a síntese dos principais resultados e conclusões.

\begin{tabular}{|c|c|c|}
\hline Estudo & Principais resultados & Conclusões \\
\hline E1 & $\begin{array}{l}\text { Os resultados revelaram que todas as } \\
\text { dimensões de QV foram } \\
\text { estatisticamente inferiores no grupo de } \\
\text { idosos com DA. Estes, quando } \\
\text { comparados ao grupo sem DA, eram } \\
\text { significativamente menos ativos, não } \\
\text { fazendo nenhum tipo de atividade física, } \\
\text { carecendo de estratégias não } \\
\text { farmacológicas de enfrentamento e } \\
\text { acesso a serviços alternativos, como } \\
\text { terapia ocupacional, fisioterapia e } \\
\text { tratamentos psicológicos. }\end{array}$ & $\begin{array}{l}\text { Os idosos com DA apresentam } \\
\text { medidas de QV inferiores, } \\
\text { sugerindo que a DA influencia } \\
\text { negativamente sua percepção. } \\
\text { Sugere-se atenção por parte } \\
\text { das políticas públicas para a } \\
\text { disponibilidade de estratégias } \\
\text { relacionadas à educação, } \\
\text { exercícios físicos, reabilitação } \\
\text { cognitiva, atividades sociais e } \\
\text { artísticas, sob a perspectiva de } \\
\text { cuidado com as dimensões } \\
\text { percebidas negativamente por } \\
\text { esta população. }\end{array}$ \\
\hline E2 & $\begin{array}{l}\text { Os escores de qualidade de vida } \\
\text { apresentaram-se diretamente } \\
\text { proporcionais a renda familiar média, ao } \\
\text { nível de instrução e a percepção de } \\
\text { suporte familiar. A medida que o nível } \\
\text { de vulnerabilidade social aumenta, } \\
\text { diminuem os escores medianos de } \\
\text { percepção de suporte familiar e de } \\
\text { qualidade de vida. }\end{array}$ & $\begin{array}{l}\text { A qualidade de vida dos idosos } \\
\text { está potencialmente sob risco } \\
\text { não apenas porque existem } \\
\text { perdas fisiológicas com o } \\
\text { passar do tempo, mas em } \\
\text { virtude da maior condição de } \\
\text { vulnerabilidade decorrente de } \\
\text { baixa escolaridade e condições } \\
\text { adversas do meio físico, social } \\
\text { ou de questões afetivas. Os } \\
\text { vínculos emocionais e as } \\
\text { relações familiares não são } \\
\text { neutros e uma boa percepção } \\
\text { de suporte familiar fomenta a } \\
\text { qualidade de vida. }\end{array}$ \\
\hline E3 & $\begin{array}{l}\text { Em relação à QV dos idosos, os } \\
\text { cuidadores consideraram desfavoráveis: } \\
\text { memória, capacidades de realizar } \\
\text { tarefas, capacidade para atividades de } \\
\text { lazer, saúde física, disposição, humor, }\end{array}$ & $\begin{array}{l}\text { Diante dos resultados, conclui- } \\
\text { se pela necessidade de uma } \\
\text { assistência que beneficie não só } \\
\text { o doente, mas também o } \\
\text { cuidador, uma vez que este }\end{array}$ \\
\hline
\end{tabular}




\begin{tabular}{|c|c|c|}
\hline & $\begin{array}{l}\text { amigos, casamento e a vida em geral. } \\
\text { Notou-se também, que as percepções } \\
\text { positivas em relação à QV dos doentes } \\
\text { de Alzheimer valorizavam aspectos } \\
\text { como família, moradia e dinheiro, pois } \\
\text { os cuidadores eram membros da família, } \\
\text { residiam juntos e se consideravam bons } \\
\text { para com seus idosos tanto nos } \\
\text { cuidados como na situação financeira } \\
\text { em que viviam. }\end{array}$ & $\begin{array}{l}\text { estando com uma QV } \\
\text { satisfatória garantirá melhor } \\
\text { assistência ao idoso e } \\
\text { consequentemente uma } \\
\text { melhoria em sua QV. O } \\
\text { cuidador é fundamental para a } \\
\text { pessoa idosa no enfrentamento } \\
\text { da dependência que a DA } \\
\text { impõe. Portanto, ações dos } \\
\text { profissionais voltadas ao bem- } \\
\text { estar deste grupo podem ajudá- } \\
\text { los a vencer as dificuldades } \\
\text { inerentes a esse processo. }\end{array}$ \\
\hline E4 & $\begin{array}{l}\text { Com relação aos sintomas depressivos, } \\
\text { foi possível notar uma melhora nos } \\
\text { pacientes que passaram por } \\
\text { treinamento e uma piora nos pacientes } \\
\text { que não participaram de nenhum tipo de } \\
\text { programa regular e sistematizado de } \\
\text { estimulação motora. } \\
\text { A intensificação destes distúrbios com a } \\
\text { progressão da doença pode representar } \\
\text { a perda da funcionalidade do paciente. } \\
\text { O programa de exercícios apresenta } \\
\text { efeitos positivos tanto na redução dos } \\
\text { transtornos depressivos com impacto } \\
\text { sobre uma melhora significativa na } \\
\text { percepção da QV tanto do paciente } \\
\text { quanto do seu cuidador. }\end{array}$ & $\begin{array}{l}\text { Conclui-se que programa de } \\
\text { exercícios físicos generalizados, } \\
\text { realizados de maneira regular e } \\
\text { sistematizada constituem uma } \\
\text { alternativa possível, de baixo } \\
\text { custo e eficaz na redução de } \\
\text { sintomas depressivos de idosos } \\
\text { com DA. }\end{array}$ \\
\hline E5 & $\begin{array}{l}\text { Estratégias não farmacológicas de } \\
\text { tratamento também devem ser utilizadas } \\
\text { com os medicamentos visando diminuir } \\
\text { a utilização, dose e efeitos colaterais, } \\
\text { além de aumentar a qualidade de vida } \\
\text { dos pacientes com DA, pois estas } \\
\text { melhoram os sintomas comportamentais } \\
\text { e psicológicos da demência. } \\
\text { A reabilitação cognitiva } \\
\text { multidisciplinar, a arteterapia, as } \\
\text { técnicas de reminiscência, a } \\
\text { estimulação cognitiva e reabilitação } \\
\text { neuropsicológica mostraram aumento na } \\
\text { QV após o tratamento. }\end{array}$ & $\begin{array}{l}\text { Os tratamentos não } \\
\text { farmacológicos mais utilizados } \\
\text { nos últimos dez anos para a } \\
\text { melhora da QV de idosos com } \\
\text { DA, de acordo com esta } \\
\text { revisão, foram à reabilitação } \\
\text { cognitiva/neuropsicológica. }\end{array}$ \\
\hline
\end{tabular}

De acordo com o $E 1$, as pessoas com DA têm vivido em condições adversas, no que se refere ao acesso a recursos de empoderamento e seus níveis quantificados de QV estão aquém da média dos idosos sem DA. Por este motivo, o estímulo e fortalecimento de parcerias entre familiares e profissionais mostram-se importantes para o bem-estar do idoso, favorecendo intervenções que garantam a conquista do envelhecimento por meio da ressignificação dos valores negativos 
associados à demência e à velhice, adequando a atenção ao paciente e reduzindo gastos públicos com cuidados (INOUYE et al., 2010).

Segundo o E2, rendas inferiores podem limitar o acesso das pessoas aos cuidados alimentares, sociais e de saúde, comprometendo de forma significativa a qualidade de vida, já as relações sociais constituem aspectos fundamentais para o bem-estar dos seres humanos e corroboram a função protetora da boa percepção de suporte familiar no fomento da qualidade de vida, desvinculando o suporte familiar do número de interações ou de pessoas dividindo o mesmo espaço, refletindo a importância da qualidade e não da quantidade ou configuração dos relacionamentos familiares (INOUYE et al., 2010).

Os dados do E3 geram questionamentos acerca das condições de vida e acesso ao serviço público de saúde das famílias menos favorecidas economicamente, supondo que estas famílias enfrentam maiores dificuldades para cuidar de seu familiar e ter acesso aos serviços de saúde. Com isso, faz-se necessário que os profissionais viabilizem propostas de intervenção que valorizem as condições de vida e de acesso aos serviços de assistência e que as mesmas sejam condizentes com as necessidades específicas desta população (BORGHI et al., 2011).

O E4 defende a prática de atividade física regular como um benefício, pois o paciente depressivo, envolvido com esta prática, pode ter como resultado o "feedback" positivo de outras pessoas aumentando a sua autoestima e reforça que o ato de exercitar-se pode servir como uma distração de pensamentos negativos e o domínio de novos hábitos podem ser importantes (BORGHI et al., 2011).

O E5 ressalta que fatores de naturezas psicológica, biológica e socioestrutural são importantes na percepção da qualidade de vida, e considera as intervenções multidisciplinares mais adequadas ao tratamento de DA, pois uma vez que não há cura ou modos de regredir a doença, o tratamento multidisciplinar do idoso com DA oferece benefícios e melhoras no estado geral do paciente (CARVALHO; MAGALHAES; PEDROSO, 2016).

Apesar de sabermos que as demências senis serão as doenças do século e que estas devem ser levadas tão a sério quanto o início da epidemia da AIDS, a presente revisão teve limitações quanto à disponibilidade de artigos redigidos em português e que abordassem a QV dos idosos, pois das publicações encontradas, a maioria eram voltadas à QV dos cuidadores.

A análise dos artigos evidenciou que o bom suporte familiar e socioeconômico, junto à prática de exercícios físicos, tarefas e atividades de lazer, participação em programas assistenciais multidisciplinares de reabilitação cognitiva e neuropsicológica que envolva o paciente e o cuidador, a arteterapia e as técnicas de reminiscência favorecem o bem-estar e melhoram a qualidade de vida de idosos com DA.

\section{CONCLUSÃO}

Os resultados alcançados nesta revisão mostraram que fatores como bom suporte familiar e socioeconômico junto à prática de exercícios físicos, tarefas e atividades de lazer, participação em programas assistenciais multidisciplinares de reabilitação cognitiva e neuropsicológica que envolva o paciente e o cuidador, a arteterapia e as técnicas de reminiscência, contribuem para a qualidade de vida de idosos com DA.

Desta forma, o enfermeiro precisa conhecer os fatores que influenciam na qualidade de vida do idoso com DA para que, junto à equipe multidisciplinar, realize atividades complementares ao tratamento farmacológico, que valorizem as 
necessidades de cada paciente e contribuam para a minimização das incapacidades provocadas pela doença.

Vale ressaltar a necessidade de mais estudos que investiguem fatores que melhoram a qualidade de vida de idosos com a DA, a fim de subsidiar novas terapêuticas que beneficiem o paciente e seus familiares.

\section{REFERÊNCIAS}

APRAHAMIAN, I.; MARTINELLI, J. E.; YASSUDA, M. S. Doença de Alzheimer: revisão da epidemiologia e diagnóstico. Rev Bras Clin Med. v. 7, n. 6, p. 27-35, 2009. Disponível em: <http://files.bvs.br/upload/S/1679-1010/2009/v7n1/a27-35.pdf>. Acesso em 01 de outubro de 2017.

ASSOCIAÇÃO BRASILEIRA DE ALZHEIMER (ABRAz). O que é Alzheimer? 2017. Disponível em: http://www.abraz.org.br/sobre-alzheimer/o-que-e-alzheimer. Acesso em: 02 de outubro de 2017.

BORGHI A. C.; SASSÁ A. H.; MATOS, P. C. B. de; DECESARO, M. das N.; MARCON, S. S. . Qualidade de vida de idosos com doença de Alzheimer e de seus cuidadores. Rev. Gaúcha Enferm. Porto Alegre, v. 32, n. 4, p. 751-758, Dec. 2011. Available from <http://www.scielo.br/scielo.php?script=sci_arttext\&pid=S198314472011000400016\&lng=en\&nrm=iso >. access on 01 Oct. 2017. http://dx.doi.org/ 10.1590/S1983-14472011000400016.

CARVALHO, P. D. P.; MAGALHAES, C. M. C.; PEDROSO, J. da S. Tratamentos não farmacológicos que melhoram a qualidade de vida de idosos com doença de Alzheimer: uma revisão sistemática. J. bras. psiquiatr. [online]. vol.65, n.4, pp.334339. ISSN 0047-2085. 2016. Disponível em: <http://www.scielo.br/pdf/jbpsiq/ v65n4/0047-2085-jbpsiq-65-4-0334.pdf>. Acesso em: 31 de maio de 2017.

FECHINE, B. R. A.; TROMPIERI, N. O processo de envelhecimento: as principais alterações que acontecem com o idoso com o passar dos anos. InterSciencePlace. v. 1, n. 20, 2015. Disponível em: <http://www.fonovim.com.br/arquivos/ 534ca4b0b3855f1a4003d09b77ee4138-Modifica----es-fisiol--gicas-normais-nosistema-nervoso-do-idoso.pdf>. Acesso em: 07 de abril de 2017.

GROPPO, H. S.;NASCIMENTO, C. M.; STELLA, F.; GOBBI, S.; OLIANI, M. M. Efeitos de um programa de atividade física sobre os sintomas depressivos e a qualidade de vida de idosos com demência de Alzheimer. Rev. bras. educ. fís. Esporte. São Paulo, v. 26, n. 4, p. 543-551, dez. 2012. Available from $<$ http://www.scielo.br/scielo.php?script=sci_arttext\&pid=S1807-55092012000 400002\&lng=pt\&nrm=iso>. Acesso: n 30 set. 2017. http://dx.doi.org/10.1590/S180755092012000400002.

INOUYE, K.; PEDRAZZANI, E. S.; PAVARINI, S. C. L. Influência da doença de Alzheimer na percepção de qualidade de vida do idoso. Rev. esc. enferm. USP, São Paulo, v. 44, n. 4, p. 1093-1099, dez. 2010. Available from <http://www.scielo.br/ scielo.php?script=sci_arttext\&pid=S0080-62342010000400034\&Ing=pt\&nrm=iso > . Acesso: n 30 set. 2017. http://dx.doi.org/10.1590/S0080-62342010000400034. 
INOUYE, K.; BARHAM, E.J.; PEDRAZZANI, E.S.; PAVARINI, S.C.I. Percepções de suporte familiar e qualidade de vida entre idosos segundo a vulnerabilidade Social. Psicol. Reflex. Crit. Porto Alegre, v. 23, n. 3, p. 582-592, 2010. Available from $<$ http://www.scielo.br/scielo.php?script= 79722010000300019\&Ing=pt\&nrm=iso>. Acesso: n 01 out. 2017. http://dx.doi.org/10.1590/S0102-79722010000300019.

Organização Mundial de Saúde (OMS). Demência: número de pessoas afetadas triplicará nos próximos 30 anos. 07 de dezembro de 2017. Disponível em: http://www.paho.org/bra/index.php?option=com_content\&view=article\&id=5560:deme ncia-numero-de-pessoas-afetadas-triplicara-nos-proximos-30-anos\&ltemid=839 acessado em: 02 de maio de 2018

SOARES, C. B.; HOGA, L.A.K. ; PEDUZZI, M.; SANGALETI,C.; YONEKURA, T.; SILVA, D.R.A.D. Revisão integrativa: conceitos e métodos utilizados na enfermagem. Revista da Escola de Enfermagem da USP. v. 48, n. 2, 2014. Disponível em: <http://www.scielo.br/pdf/reeusp/v48n2/pt_0080-6234-reeusp-48-02335.pdf >. Acesso em: 28 de setembro de 2017.

TALMELLI, L. F. da S.; VALE, F.de A. C. do; GRATÃO, A.C.M.; KUSUMOTA, L.;RODRIGUES, R.A.P. Doença de Alzheimer: declínio funcional e estágio da demência. Acta paul. enferm. São Paulo, v. 26, n. 3, p. 219-225, 2013. Available from <http://www.scielo.br/scielo.php?script=sci_arttext\&pid=S0103-2100201300 0300003\&lng=en\&nrm=iso>. Acesso: n 03 Out. 2017. http://dx.doi.org/10.1590/ S0103-21002013000300003. 